\title{
Modeling an Energy-Dispersive X-ray Diffraction System for Drug Detection
}

\author{
Silvia Pani, Emily Cook, Julie Horrocks, Leah George, Sheila Hardwick and Robert Speller
}

\begin{abstract}
A system for drug detection using X-ray diffraction is currently being developed by the DILAX collaboration. A simulation program for modeling the response of an energydispersive $\mathrm{X}$-ray diffraction system has been developed, with the two-fold aim of selecting possible configurations prior to experimental tests and of generating data for statistical models for prediction of drug content.

Simulated data showed a good agreement with experimental results. The data showed that the main factor affecting the shape of the diffraction pattern is the thickness of the sample. Scatter angle and detector energy resolution have a smaller effect on the diffraction pattern. This suggests that cheaper, roomtemperature detectors can be used for a drug detection system without any loss in sensitivity and specificity.
\end{abstract}

Index Terms-Nondestructive testing, X-ray imaging, Diffraction, Geometric modeling

\section{INTRODUCTION}

$\mathrm{T}$ he potential of X-ray diffraction (XRD) for material identification in different fields has been demonstrated by several authors [1], [2]. This is because XRD patterns, resulting from interference of coherently scattered X-rays, are dependent upon the inter-atomic and inter-molecular distances of the material inspected, and hence are characteristic of each material.

In particular, it has been shown that energy-dispersive X-ray diffraction (EDXRD) combined with multivariate analysis for drug identification allows illegal drug identification [3]. The DILAX project is aimed at looking at the best options for a system for illicit drug identification using X-ray diffraction.

In order to do so, several combinations of geometries and X-ray spectra will be experimentally evaluated. A program has been built to simulate diffraction patterns of a range of materials in order to pre-select a limited number of combinations. The aim of the program is also to generate the large amounts of data required to build up multivariate analysis models for prediction of drug content.

Manuscript received

S. Pani and J. Horrocks are with Clinical Physics, School of Medicine and Dentistry, Queen Mary, University of London and Barts and The London NHS Trust, London, UK (tel 0044207601 8292; fax 0044207601 8294, emails.pani@qmul.ac.uk)

E. Cook and R. Speller are with the Department of Medical Physics and Bioengineering, University College London, London, UK.

L. George and S. Hardwick are with the Counter Drug Technologies Team, Home Office Scientific Development Branch, Sandridge, UK.

\section{MEthodS}

\section{A. The system}

A set of modelling routines has been developed in IDL (ITT Visual Information Systems) for calculating the alterations to high-resolution diffraction patterns caused by:

a) Shape of the X-ray beam spectrum;

b) Blurring caused by the finite energy resolution of the detector;

c) Blurring caused by the finite angular resolution of the system;

d) Attenuation within the sample itself.

A schematic diagram of the system modeled is shown in Figure 1.

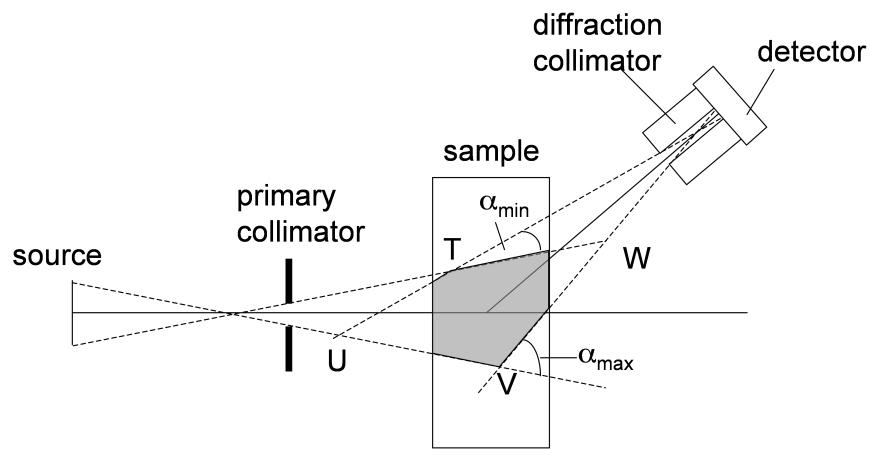

Figure 1. Schematic drawing of the energy-dispersive diffractometer modeled. The shaded area shows the effective scatter volume due to sample size, as opposed to the total scatter volume delimited by the vertices T, U, V, W.

A collimated X-ray beam from a conventional source irradiates a sample. A spectroscopic detector is placed at an angle $\vartheta$ from the beam axis. A scatter collimator, placed in front of the detector, limits the range of the scatter angles detected. The centre of the diffractometer is defined as the intersection between the scatter collimator axis and the beam axis.

The user-defined parameters are the following:

- Geometrical parameters of the diffractometer (source size and profile, scattering angle $\vartheta$, distance between source and primary beam collimator, distance between primary beam collimator and centre of diffractometer, primary collimator aperture, scatter collimator aperture, scatter collimator length).

- Sample properties (thickness, number of layers, position with respect to the centre of the diffractometer, 
composition).

- X-ray beam spectrum.

- Detector energy resolution.

The starting point for the simulation are high-resolution diffraction patterns of drugs, cutting agents and possible packaging as obtained from a high resolution angle-dispersive diffractometer (Panalytical X'Pert Pro) or from the ICDDJCPDS database [4]. These patterns are recorded as a function of the momentum transfer, $\chi=1 / \lambda \sin (\vartheta / 2)$, where $\lambda$ is the $\mathrm{X}$ ray wavelength, and are evenly sampled in $\chi$.

They are weighted for the X-ray beam spectrum and then convolved for two functions describing the energy and the angular resolution of the system. Finally, corrections for sample attenuation are applied to the resulting pattern.

\section{B. Weighting for beam spectrum and convolution by detector energy resolution}

$\mathrm{X}$-ray spectra are generated using the IPEM catalogue [5].

Weighting for X-ray beam spectrum is done by converting the energy-axis of the spectrum into momentum transfer by means of the following equation:

$\chi\left(\mathrm{nm}^{-1}\right)=0.806 \cdot E(\mathrm{keV}) \cdot \sin (\vartheta / 2)$

The spectrum is then resampled using linear interpolation to match the sampling of the original diffraction pattern.

In a similar way, the function representing the energy resolution of the detector (a Gaussian with a user-specified FWHM, or an experimental curve) is converted into momentum transfer resolution and sampled with the same step as the diffraction pattern. The weighted diffraction pattern is then convolved by this function.

\section{Convolution by angular resolution}

The angular resolution results from a combination of the geometrical parameters of the system and of the sample. As shown in Fig. 1, the scattering volume, delimited by the vertices $T, U, V, W$, is defined by the intersection of the collimated primary beam with the angular acceptance of the scatter collimator. If the sample is contained within the scatter volume, its vertices are modified accordingly (grey area).

The angular resolution can be represented as a histogram of the scattering angles achieved from all points of the effective scatter volume. To compute it, the source aperture is divided into segments. Assuming a uniform angular distribution for photons emitted from the generic point $\mathrm{P}$ within the source, the range of angles allowing a photon to cross the primary collimator is calculated and divided into equal angular intervals. The length of the scatter volume is in turn divided into intervals. This divides the area of the sample seen by the point $\mathrm{P}$ into pixels (see Fig. 2).

For each pixel, the range of possible angles under which the scatter collimator aperture is seen is calculated; the difference from the angle at which the photon has been generated by the source is calculated, giving the net scatter angle; weighting for pixel size is applied and a histogram of scatter angles is built. The angular resolution is calculated by repeating this procedure for each segment of the source. Figure 3 shows examples of the angular resolution for samples of different thicknesses and with the same geometrical parameters for the diffractometer.

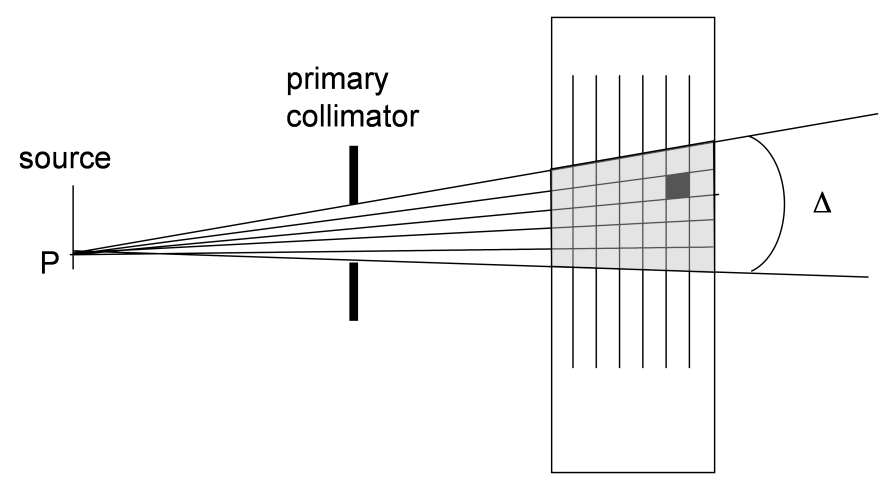

Figure 2. Schematic diagram showing the subdivision into pixel of the portion of sample irradiated by the generic source point $P$.

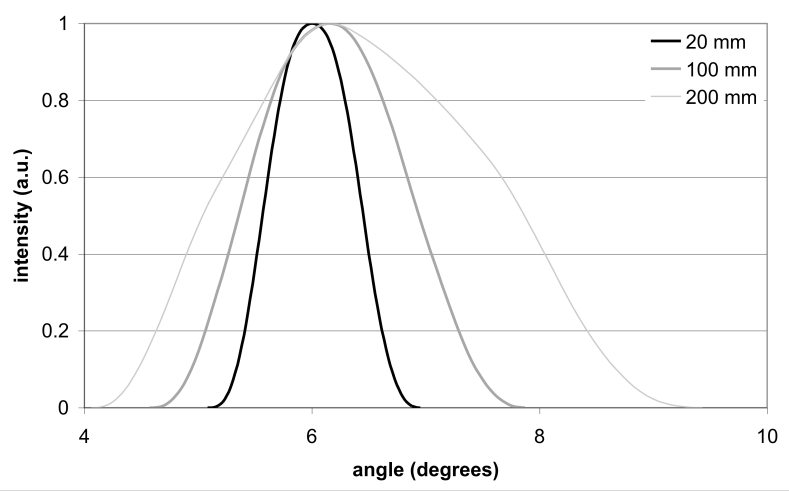

Figure 3. Angular resolution for samples of different thicknesses in the centre of the scatter volume. The geometrical parameters of the system are: collimator angle $6^{\circ}$; source-primary collimator distance $300 \mathrm{~mm}$; primary collimator-centre of diffractometer distance $200 \mathrm{~mm}$; centre of diffractometerscatter collimator distance $338 \mathrm{~mm}$; source size $3 \mathrm{~mm}$; primary collimator aperture $1 \mathrm{~mm}$; scatter collimator aperture $1.1 \mathrm{~mm}$; scatter collimator length $20 \mathrm{~mm}$.

For the present application, the source was divided into 100 segments; the angular interval for dividing the scatter area into pixels was $1 \mathrm{mrad}$ and the depth of the pixels was $10 \mu \mathrm{m}$.

In order to convolve the diffraction patterns by the angular resolution, the latter cannot be assumed to be constant. As it can be seen from Equation 1, momentum transfer is a nonlinear function of the angle, and a variation in the angle implies a non-constant variation of the momentum transfer over the momentum transfer range. Hence, convolution by angular aperture is performed as follows: for each component $\chi_{j}$ of the diffraction pattern, the x-axis (angle) of the angular resolution is converted into momentum transfer as:

$\bar{\chi}=\chi_{j} \frac{\sin (\bar{\vartheta} / 2)}{\sin (\vartheta / 2)}$

where $\bar{\vartheta}$ is the generic value of the $\mathrm{x}$-axis for the angular resolution.

The previously obtained diffraction pattern is convolved by the angular resolution and the $j$-th value is extracted. This will form the $j$-th component of the final diffraction pattern. The procedure is repeated for each value of $\chi_{j}$. 
In the case of a multi-layer sample, the procedures of weighting by beam spectrum, convolution by energy resolution, calculation of, and convolution by, the angular resolution are carried out separately for each layer. The convolved patterns are finally summed up to form the total convolved pattern $T$.

\section{Attenuation corrections}

A simplified approach is used to calculate the correction for the attenuation of X-rays within the sample.

The number of photons of energy $E$ scattered by a layer of thickness $d x$ at a depth $x$ inside the sample and reaching the detector is given by (see Fig. 4)

$d N_{\text {det }}(E)=N(E, x) \cdot \mu_{s}(\chi, x) \cdot e^{-\int_{D} \mu(\xi) d \xi} d x$

Here $N(E, x)$ is the number of photons of energy $E$ reaching a depth $x$ within the sample, $\mu_{s}(\chi, x)$ is the linear differential scatter coefficient at a depth $x$ and for a momentum transfer $\chi$ $=0.806 E \sin (\vartheta / 2)$, and the exponential term accounts for the attenuation along the remaining path length $D$ within the sample, $\mu(\xi)$ being the linear attenuation coefficient at the generic point $\xi$.

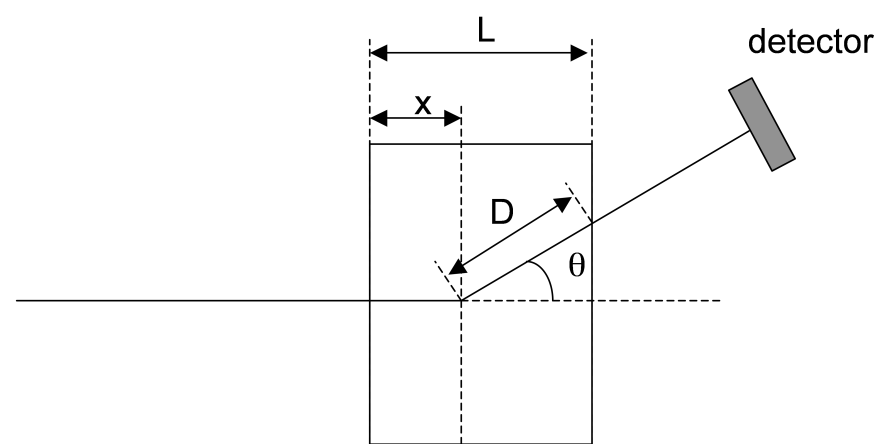

Figure 4. Simplified geometry used for calculating attenuation corrections.

If the angle $\vartheta$ is sufficiently small, the approximation $D \cong D$ $\cos (\vartheta)$ holds, and, if the variations in sample composition along the vertical axis are negligible, the integral of the linear attenuation coefficient along the path $D$ can be approximated by that from $x$ to $L$.

Hence, if $N_{0}(E)$ is the number of photons of energy $E$ incident on the sample, we have

$$
\begin{aligned}
& d N_{\mathrm{det}}(E)=N_{0}(E) \cdot e^{-\int_{0}^{x} \mu(\xi) d \xi} \cdot \mu_{s}(\chi, x) \cdot e^{-\int_{x}^{L} \mu(\xi) d \xi} d x= \\
& =N_{0}(E) \cdot \mu_{s}(\chi, x) \cdot e^{-\int_{0}^{L} \mu(\xi) d \xi} d x
\end{aligned}
$$

Under the above assumptions, the exponential term accounts for the attenuation within the sample regardless of the position of the scattering point considered, and can hence be applied "a posteriori" to the total convolved diffraction pattern. Moreover, for a finite number of layers, each one with a constant composition, the integral can be approximated by a discrete sum. Hence, the $j$-th element of the total diffraction pattern corrected for sample attenuation is

$T_{j}^{\prime}=T_{j} \cdot e^{-\sum_{i} \mu_{i}\left(E_{j}\right) L_{i}}$

where $L_{i}$ is the thickness of the $i$-th layer of the sample, and
$\mu_{i}\left(E_{j}\right)$ is the linear attenuation coefficient for the $i$-th material at an energy $E_{j}=\chi_{j} /(0.806 \sin (\vartheta / 2))$.

\section{RESULTS}

\section{A. Comparison with experimental data}

The validity of the model was tested against experimental data for different geometries.

A HPGe detector was used in all cases, with an energy resolution of $0.7 \mathrm{keV}$ at $59.5 \mathrm{keV}$. The diffractometer centrescatter collimator distance was $338 \mathrm{~mm}$; the source-primary collimator distance was $300 \mathrm{~mm}$; the primary collimator aperture was $0.5 \mathrm{~mm}$; the scatter collimator length was 20 $\mathrm{mm}$.

The X-ray tube had a $\mathrm{W}$ anode and $1 \mathrm{~mm}$ Be inherent filtration, and was operated at $80 \mathrm{kVp}$; the source size was 3 $\mathrm{mm}$. These parameters were input to the IPEM database [5] for generating the spectrum used in the simulations. The sample used was a thin plastic tube, $5 \mathrm{~mm}$ in diameter, containing Zirtek ${ }^{\circledR}$, a medication featuring diffraction peaks in the same region as some illegal drugs.

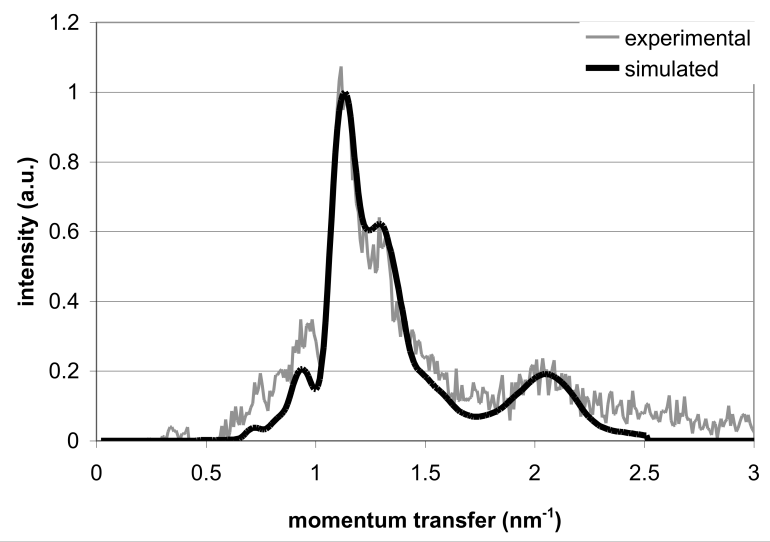

Figure 5. Experimental vs simulated data for a Zirtek ${ }^{\circledR}$ sample. The diffraction collimator aperture was $2 \mathrm{~mm}$; the scatter angle is $7^{\circ}$.

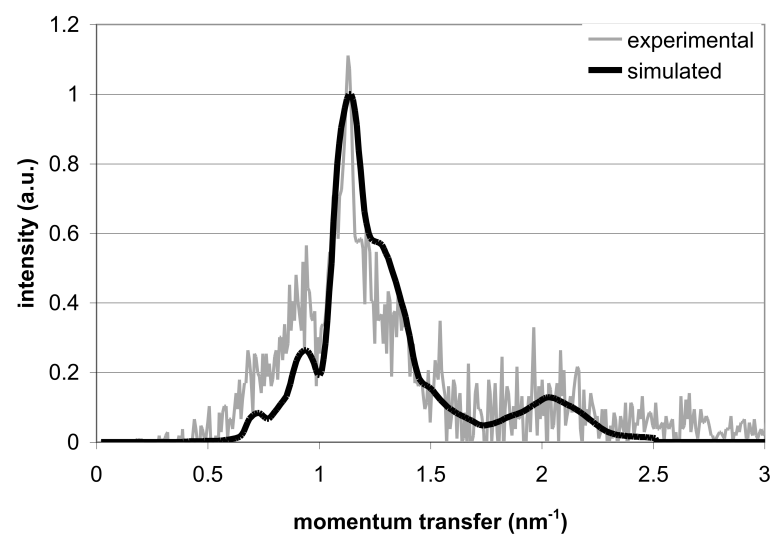

Figure 6. Experimental vs simulated data for a Zirtek ${ }^{\circledR}$ sample. The diffraction collimator aperture was $1 \mathrm{~mm}$; the scatter angle is $6^{\circ}$.

Fig. 5 and Fig. 6 show comparison between experimental data and simulated data. The discrepancy for momentum transfer values below $1 \mathrm{~nm}^{-1}$ could result from a mismatch between the actual tube filtration and the nominal one. This has a bigger effect on the low-energy, and hence low- 
momentum transfer, part of the spectrum. On the other hand, the height and width of the experimental diffraction peaks are correctly reproduced in the simulation. This confirms that the simulations give a realistic description of the signal alteration caused by geometrical and spectral parameters, as well as sample and detector properties.

\section{B. Effect of different parameters on final patterns}

Fig. 7 - Fig. 9 show results of the simulation when different parameters are changed. It is clear from the graphs that the parameter that most strongly affects the shape of the diffraction pattern is sample thickness (Fig. 7). This causes variations in the peak width, due to the dependence of angular resolution upon sample thickness (see Fig. 3), and shifts towards the high momentum-transfer (high-energy) part of the diffraction pattern in thicker samples, due to beam hardening when crossing the sample. On the other hand, changing the scatter angle detected does not significantly affect the shape and the resolution of the diffraction patterns (Fig. 8).

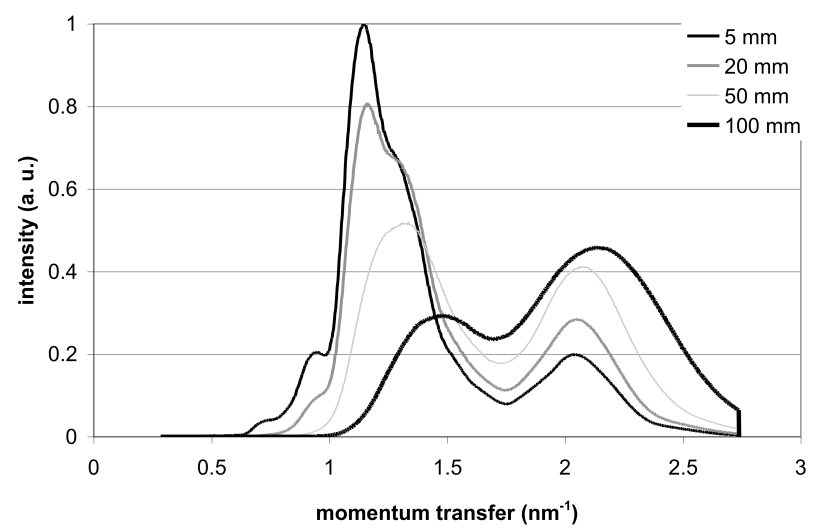

Figure 7. Effect of sample thickness on the diffraction pattern for a Zirtek ${ }^{\circledR}$ sample. The remaining parameters are: scatter angle $6^{\circ}$; source size $3 \mathrm{~mm}$; source-primary collimator distance $300 \mathrm{~mm}$; primary collimator aperture 1 $\mathrm{mm}$; primary collimator-centre of diffractometer distance $200 \mathrm{~mm}$; centre of diffractometer-scatter collimator distance $338 \mathrm{~mm}$; scatter collimator length $20 \mathrm{~mm}$; scatter collimator aperture $1.1 \mathrm{~mm}$; detector energy resolution 0.7 keV FWHM.

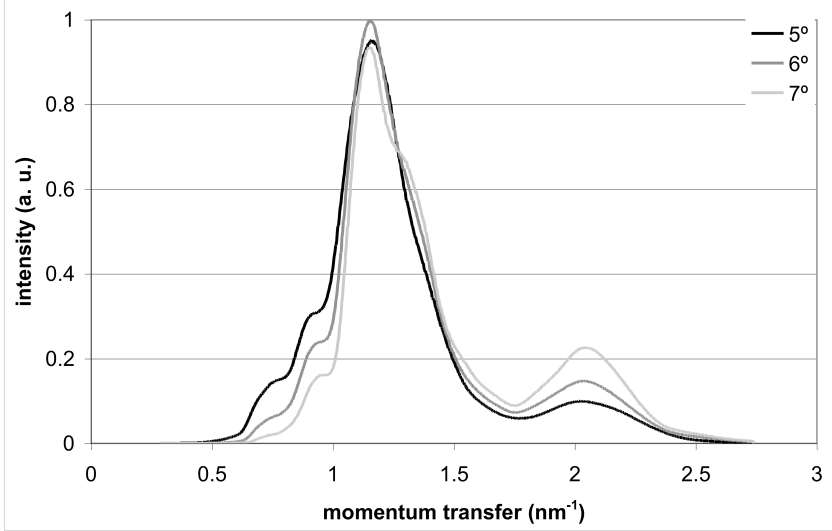

Figure 8. Effect of scatter angle on the diffraction pattern for a $10 \mathrm{~mm}$ thick Zirtek ${ }^{\circledR}$ sample. The remaining parameters are those specified in Fig. 7.

Moreover, the energy resolution of the detector does not alter significantly the shape of the diffraction pattern. Fig. 9 shows comparison between the diffraction patterns obtained with a constant $0.7 \mathrm{keV}$ energy resolution (typical value for a
HPGe detector) and with a constant $4 \mathrm{keV}$ energy resolution (typical value for a CZT detector).

This is because for the typical geometries adopted, allowing acceptable counting statistics, the angular resolution is predominant compared to the energy resolution.

Results from statistical analysis [6] confirmed that the predictory power of a CZT-based system is not lower than that of a HPGe-based system.

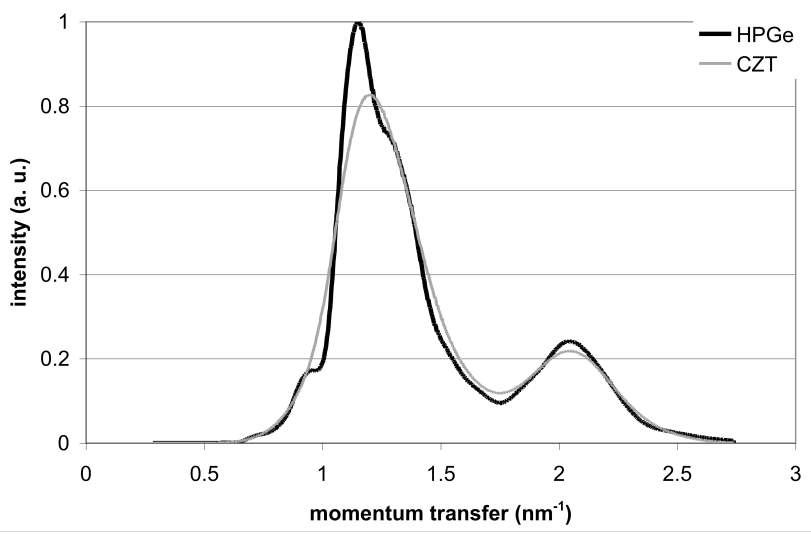

Figure 9. Effect of detector energy resolution on the diffraction pattern for a $10 \mathrm{~mm}$ thick Zirtek ${ }^{\circledR}$ sample. The remaining parameters are those specified in Fig. 7.

\section{CONCLUSIONS}

A simulation program has been developed to model an energy-dispersive X-ray diffraction system for drug identification.

The effect of different parameters on the shape of the final diffraction pattern has been evaluated, showing that sample thickness is the dominant factor. Models for statistical analysis will be developed to take this factor into account.

On the other hand, because the angular resolution is predominant, the effect of energy resolution on the diffraction pattern is not significant. This result is particularly encouraging in that it suggests that cheaper room-temperature detectors, such as CZT, offer a valid alternative to highresolution detectors such as HPGe for the development of a system for drug characterization. Different geometries will be tested in order to optimize the predictory power/acquisition time trade-off.

\section{REFERENCES}

[1] R. D. Luggar, J. A. Horrocks, R. D. Speller and R. J. Lacey, "Low angle X-ray scatter fro explosives detection: a geometry optimization". Applied Radiation and Isotopes, vol. 48(2), pp. 215-224, 1998.

[2] P. C. Johns and M. J. Yaffe, "Coherent scatter in diagnostic radiology" Med. Phys., vol. 10(1), pp. 40-50, 1983.

[3] E. Cook, R. Fong, J. Horrocks, D. Wilkinson and R. Speller, "Energy dispersive X-ray diffraction as a means to identify illicit materials". Applied Radiation and Isotopes, vol. 65(8), pp. 959-967, 2007.

[4] S. N. Kabekkodu, J. Faber, and T. Fawcett, "New powder diffraction file (PDF-4) in relational database format: advantages and data-mining capabilities". Acta Crystallographica B, vol. 58, pp. 333-337, 2002.

[5] K Cranley, B J Gilmore, G W A Fogarty, and L Desponds, "Catalogue of diagnostic X-ray spectra and other data". IPEM, 2002, on CD-ROM.

[6] E. J. Cook, S. Pani, L. George, S. Hardwick, J. A. Horrocks, and R. D. Speller, "Multivariate data analysis for drug identification using energydispersive X-Ray diffraction". Submitted to IEEE Trans. Nucl.Sci. 